\title{
Avaliação de políticas e iniciativas públicas de segurança alimentar e nutricional: dilemas e perspectivas metodológicas
}

\author{
Evaluation of public policies and initiatives in food and nutrition \\ security: dilemmas and methodological perspectives
}

Abstract The scope of this article is to analyze the main challenges and perspectives linked to the evaluation of public policies in food and nutrition security. The conclusion reached is that considering the complexity of actions in this area, it is important to discuss the limits of traditional evaluation strategies and move forward with the creation of new theoretical and methodological alternatives.

Key words Evaluation, Methodology, Public policies, Food and nutrition security
Resumo O objetivo do presente artigo é analisar os principais desafios e perspectivas da avaliação de politicas e iniciativas públicas voltadas à segurança alimentar e nutricional. Conclui-se que devido à complexidade das ações na área é importante discutir os limites das estratégias avaliativas tradicionais e avançar na construção de novas abordagens teóricas e metodológicas.

Palavras-chave Avaliação, Metodologia, Politicas públicas, Segurança alimentar e nutricional 


\section{Introdução}

Ao longo das últimas décadas o debate sobre a avaliação das políticas públicas de alimentação e nutrição atraiu planejadores, pesquisadores e estudiosos de diferentes áreas e disciplinas. Já na primeira metade do século XX, o médico e sociólogo Josué de Castro ${ }^{1}$ contribuiu de maneira inquestionável para a análise da fome como expressão das relações entre o homem e o meio ambiente chamando a atenção para os limites da visão do desenvolvimento baseada apenas no crescimento econômico. Após a II Guerra Mundial sucessivas crises no abastecimento de alimentos e o agravamento do quadro de fome e da miséria no mundo impulsionaram a criação da Organização das Nações Unidas para a Agricultura e Alimentação (FAO), a Organização Mundial da Saúde (WHO) e o Fundo das Nações Unidas para a Infância (UNICEF). É neste período, marcado pela preocupação com a questão da disponibilidade de alimentos e com o risco de embargos econômicos em zonas de conflito, que emerge o conceito de segurança alimentar ${ }^{2}$.

A busca de alternativas para garantir o aumento da produção de alimentos e a manutenção de fluxos de armazenamento estratégicos orientou as iniciativas na área até meados dos anos 80 . No entanto, paradoxalmente, apesar do aumento da produtividade agrícola o mundo assistiu ao agravamento dos quadros de desnutrição crônica e demais formas de privação alimentar em vários países e regiões do planeta. As controvérsias em torno do tratamento dado ao tema cultivaram abordagens mais complexas e sofisticadas acerca dos desafios a serem enfrentados. Com o apoio da FAO e da OMS, a Conferência Internacional de Nutrição realizada em Roma (1992), a Conferência Internacional de Direitos Humanos (1993) e a Cúpula Mundial de Alimentação (1996) tornaram-se espaços privilegiados para a elaboração de um conceito de segurança alimentar mais abrangente baseado não só na disponibilidade, mas também no acesso a alimentos suficientes para a manutenção da vida. Frente à existência de amplas parcelas da população mundial vivendo na pobreza e às ameaças provocadas pelo desenvolvimento agroindustrial, como, por exemplo, os riscos de contaminação pelo uso indiscriminado de agrotóxicos e a expulsão dos pequenos produtores rurais, a dimensão nutricional foi integrada ao conceito de segurança alimentar. Além disso, os princípios da soberania dos povos, no que se refere à decisão sobre o que produzir e como produzir e, também, do direito humano à alimentação saudável com respeito à diversidade cultural, tornaram-se indissociáveis da concepção contemporânea de segurança alimentar e nutricional. No Brasil, a definição sistematizada pelo Conselho de Segurança Alimentar e Nutricional (CONSEA $)^{3}$ e referendada na Lei Orgânica de Segurança Alimentar e Nutricional (LOSAN) ${ }^{4}$ revela o diálogo com esta trajetória e a centralidade do tema da segurança alimentar e nutricional na agenda política:

Segurança Alimentar e Nutricional (SAN) consiste na realização do direito humano ao acesso regular e permanente a uma alimentação saudável, de qualidade e quantidade suficientes, e que não comprometa o acesso a outras necessidades essenciais e ao sistema alimentar futuro. A base da segurança alimentar e nutricional são práticas alimentares saudáveis que respeitem as diversidades culturais e sejam sustentáveis do ponto de vista socioeconômico e agroecológico, assegurando o bem-estar e a saúde dos indivíduos.

No entanto, para Lang e Heasman ${ }^{5}$, apesar do debate ter avançado ao longo do tempo ainda existe uma verdadeira batalha de paradigmas em torno dos rumos da política alimentar e nutricional. Em grande parte, isto se deve à presença de diferentes grupos de interesse envolvendo setores corporativos e grandes indústrias. De acordo com os autores, embora tradicionalmente a agricultura tenha dominado a agenda na área, cada vez mais as pressões advindas dos oligopólios e das grandes redes responsáveis pelo processamento e comercialização dos alimentos têm contribuído para mudanças no consumo alimentar. Neste cenário, muitas vezes as profundas interações entre a promoção da saúde e o perfil alimentar e nutricional assumem um caráter fortuito e aleatório apesar do crescimento da obesidade, diabetes, diferentes tipos de câncer e demais problemas associados à questão alimentar. O objetivo do artigo é analisar os principais desafios e as perspectivas da avaliação de políticas e iniciativas públicas voltadas à segurança alimentar e nutricional buscando contribuir para a construção de novas alternativas teóricas e metodológicas.

\section{Governança Alimentar e Nutricional: instituições, atores e contextos}

Apesar dos fortes interesses e conflitos econômicos ligados à produção e comercialização de alimentos, em vários países o fortalecimento de parcerias com organizações não governamen- 
tais, academia e demais setores da sociedade civil tem contribuído para a formulação de políticas de segurança alimentar e nutricional ambientalmente sustentáveis, capazes de favorecer a equidade de gênero no campo e a redução dos efeitos das mudanças climáticas na produção agrícola. No entanto, é importante destacar que o desenho de ações integradas e a construção de compromissos sólidos em torno da alimentação saudável exigem diferentes níveis de articulação intersetorial e mecanismos de coordenação política. Neste aspecto, a chamada food governance ${ }^{6}$ ou a governança alimentar e nutricional envolve o fortalecimento de canais de negociação intergovernamentais e a adoção de novos mecanismos de articulação entre instituições, mercados e múltiplos grupos de interesse locais e globais. Novos arranjos institucionais são cruciais para lidar com novas questões como, por exemplo, a emergência de alimentos geneticamente transformados, fortificados e processados tecnologicamente de diferentes maneiras e que impactam tanto a produção agrícola e industrial como o perfil das redes de comercialização e padrões de consumo.

Neste contexto, não só a quantidade, mas a qualidade dos alimentos produzidos e disponibilizados para a população devem orientar a agenda política e o desenho de instrumentos legais de regulação e fiscalização. Sabemos, porém, que normas e leis embora aprovadas e sancionadas muitas vezes enfrentam enormes dificuldades para sua efetiva implementação. Os setores que possuem fortes conexões com o agronegócio e a comercialização de pesticidas químicos, por exemplo, tendem a mobilizar recursos institucionais e organizacionais para impedir mudanças que possam atingir suas expectativas de lucro. Em muitos países o uso clandestino de substâncias perigosas afeta de maneira cumulativa o ar, a água, o solo e a saúde humana.

De acordo com o Programa de Análise de Resíduos de Agrotóxicos (PARA) da Agência Nacional de Vigilância Sanitária (ANVISA) o Brasil - maior consumidor de agrotóxicos no mundo - possui um terço dos alimentos destinados ao consumo contaminados por resíduos além dos limites aceitáveis ${ }^{7}$. A situação ainda é mais alarmante na medida em que muitas substâncias utilizadas no país são proibidas em países da Comunidade Europeia e EUA ${ }^{8}$. Embora a Lei dos Agrotóxicos ${ }^{9}$ criada em 1989 e que busca regular o controle, a inspeção e a fiscalização de agrotóxicos no país represente uma conquista importante, ainda são grandes os desafios para a construção de modelos de produção agroecológicos.

Em 2012 foi instituída a Política Nacional de Agroecologia e Produção Orgânica (PNAPO) que prevê a participação da sociedade civil e do governo no desenho e na implementação de ações voltadas à garantia da sustentabilidade da agricultura familiar. Dentre as ações propostas que integram esta intervenção destacam-se a preservação e a disseminação de sementes crioulas, ou seja, sementes produzidas pelos próprios agricultores de acordo com as possibilidades de cada contexto local e com menor dependência de produtos químicos. Nesta direção, projetos de agricultura urbana e periurbana ${ }^{10}$, que visam estimular a produção orgânica de alimentos em áreas ociosas das grandes cidades, e o Programa de Aquisição de Alimentos (PAA) ${ }^{11}$ também contribuem para o fortalecimento da agricultura familiar e a adoção de novas práticas de comercialização e abastecimento. No entanto, a hegemonia de latifundiários e de grandes empresas e o interesse no uso indiscriminado de agrotóxicos permanecem sendo fortes obstáculos para a construção de um novo modelo de produção agrícola capaz de favorecer a biodiversidade, o equilíbrio ecológico e a equidade alimentar e nutricional.

No que se refere à propaganda de alimentos, também existem resistências do setor privado às tentativas de controle e monitoramento. As barreiras para a regulação da propaganda na área impactam fortemente o alcance de estratégias mais eficazes de promoção da alimentação saudável. Frente à desestruturação da alimentação cotidiana na maioria das cidades devido à falta de tempo para preparação dos alimentos a propaganda pode reforçar ainda mais a prática do snacking e o acesso a comidas rápidas e simplificadas fora de casa ${ }^{12}$. Além disso, várias pesquisas revelam que as estratégias agressivas utilizadas na propaganda de alimentos processados e altamente calóricos contribuem para o crescimento da obesidade em adultos e, especialmente, crianças. De acordo com a American Heart Association (AHN) as crianças são expostas constantemente a propagandas de alimentos ricos em açúcar e sódio através da televisão, internet, videogames e celulares ${ }^{13}$. Um estudo realizado nos EUA revelou que cerca de $2 / 3$ dos alimentos e bebidas infantis não contêm frutas apesar de apresentarem imagens e referências a estes alimentos na embalagem $^{14}$. No Brasil, algumas iniciativas governamentais e da sociedade civil como a Frente pela Regulação da Publicidade de Alimentos que reúne diversas entidades profissionais e associa- 
ções de defesa do consumidor, segurança alimentar e nutricional e promoção da saúde têm chamado a atenção sobre os riscos relacionados ao consumo de alimentos de baixo valor nutricional e à necessidade de maior controle das campanhas publicitárias que visam ampliar a comercialização destes produtos ${ }^{15,16}$.

Nessa conjuntura, além da criação de marcos regulatórios, é preciso construir pactos e compromissos capazes de equacionar disputas e conflitos de interesse. Não se trata apenas de reforçar antagonismos entre interesses públicos e privados. Um caminho mais promissor parece ser a adoção de estratégias capazes de contornar pontos de vista discrepantes e explorar a possibilidade de novos acordos para o alcance de modelos de desenvolvimento saudável e sustentável. $\mathrm{Na}$ verdade, é importante evitar a visão dos governos como arenas homogêneas e submetidas invariavelmente a interesses privados ou às pressões da sociedade civil.

As instituições governamentais são atravessadas por controvérsias e constrangimentos políticos e revelam diferentes graus de autonomia no processo de definição de agendas e problemas prioritários. Neste sentido, é preciso reconhecer que a conformação de políticas de segurança alimentar e nutricional é um processo complexo, dinâmico e afetado por diferentes forças. Mas, sem o engajamento público em torno da alimentação como direito humano, um dos principais desafios políticos e sociais contemporâneos, a viabilidade e o impacto das iniciativas serão reduzidos. Ao mesmo tempo, sem a avaliação das experiências em cada contexto social dificilmente será possível compreender a natureza e a complexidade da questão alimentar e nutricional e avançar no desenho de novas estratégias políticas.

\section{A avaliação de políticas e programas de promoção da segurança alimentar e nutricional: perspectivas teóricas e alternativas metodológicas}

No Brasil e em vários países, a introdução da alimentação no rol dos direitos constitucionais e a aprovação de uma Lei Orgânica de Segurança Alimentar e Nutricional representam marcos consistentes do processo recente de amadurecimento conceitual e institucional na área. No entanto, como analisam Prado et al. ${ }^{17}$,junto às perspectivas intersetorial, participativa e interdisciplinar trazidas no bojo da reconfiguração da questão alimentar e nutricional também vieram novos desafios teóricos e metodológicos para a avaliação das ações.

Sabemos que na maioria das sociedades democráticas modernas o surgimento de novas demandas sociais e o maior questionamento sobre o impacto das ações dos governos impulsionaram de maneira decisiva a avaliação de políticas públicas. No Brasil, o debate sobre a avaliação de políticas e programas sociais ganhou força após o processo de redemocratização do país nos anos 80 e, em geral, o principal foco foram os resultados finais das intervenções públicas. De certa forma, esta perspectiva, como em vários países, foi apoiada pela chamada evidence based policy ${ }^{18}$, ou seja, pela preocupação com o desenho de programas e políticas públicas baseadas em evidências de efetividade, eficiência e eficácia e, basicamente, com a gestão a partir de resultados.

Sem dúvida, este esforço contribuiu para a sistematização de informações substantivas sobre os alcances das ações. No campo das políticas de alimentação e nutrição, estudos avaliativos revelaram padrões clientelistas, superposição de clientelas e estratégias ineficazes em torno da alocação de recursos ${ }^{19}$. Tais estudos apontaram ainda o baixo impacto e os resultados perversos dos programas de distribuição de alimentos que não atingiam os mais pobres apesar do altíssimo custo. No Brasil, o Programa de Alimentação do Trabalhador (PAT), voltado apenas ao setor formal do mercado de trabalho, gerou fortes desigualdades no acesso à segurança alimentar ${ }^{20}$. As evidências em torno do alcance dos programas e iniciativas foram, desta maneira, fundamentais para a fertilização da pesquisa avaliativa na área. No entanto, frente à natureza complexa, intersetorial e participativa das ações orientadas pela perspectiva da segurança alimentar e nutricional no contexto contemporâneo é importante ampliar os enfoques adotados. Embora a análise dos resultados finais dos programas de segurança alimentar e nutricional apoiadas no enfoque experimental e na avaliação somativa sejam cruciais, abordagens onde sejam tensionadas as fronteiras entre a avaliação de processos e de resultados com ênfase nos diferentes contextos locais de implementação trazem novas perspectivas e alcances.

Sabemos que as intervenções em segurança alimentar e nutricional tendem cada vez mais a mobilizar diferentes atores e parcerias com organizações estatais, comunitárias, setor privado, instituições de ensino e pesquisa e, portanto, comunidades com diferentes concepções, práticas e recursos. Além disso, dificilmente os alvos de 
programas de segurança alimentar implicam mudanças de aspectos isolados. Pelo contrário, mudanças efetivas no perfil de segurança alimentar tendem a envolver fluxos interdependentes mesmo que o foco seja o consumo ou a produção de alimentos ${ }^{21}$. Neste sentido, podemos dizer que assim como as iniciativas no campo da promoção da saúde, os programas de segurança alimentar e nutricional tensionam as concepções tradicionais de causalidade, variáveis controladas, atribuição e linearidade ${ }^{22}$. Ou seja, é difícil perceber efeitos ou impactos ligados exclusivamente a uma única intervenção exatamente porque as ações na área são por natureza complexas, multiestratégicas e intersetoriais. As iniciativas, em geral, envolvem mudanças em escolas, serviços de saúde, comunidades, meio ambiente e também em padrões econômicos e culturais. Com isso, políticas e programas são altamente dependentes do contexto de implementação interagindo fortemente com as singularidades dos respectivos contextos com destaque para as concepções, os interesses e as expectativas dos atores locais. Além disso, a maioria das experiências revelam processos decisórios mergulhados em situações de conflito e negociação. Geralmente não é possível reconhecer um passo a passo linear e etapas desenvolvidas sempre de cima para baixo - top down - ou de baixo para cima - bottom up. Neste cenário, as alternativas avaliativas capazes de favorecer a análise de processos, atores sociais e contextos ${ }^{23}$ podem contribuir para mudanças nas práticas, impactar as diferentes redes de profissionais, favorecer coalizões e parcerias e influenciar dinâmicas institucionais.

Assim, na perspectiva de compreender os contextos organizacionais e a dinâmica das relações entre os agentes dos programas e seus respectivos contextos de interação é importante identificar as possíveis discrepâncias entre o planejado e o executado. Nesta direção, a análise dos programas de segurança alimentar e nutricional baseada no exame de suas premissas teóricas, ou seja, na compreensão da cadeia de efeitos que liga recursos, atividades, resultados intermediários e metas a serem alcançadas, pode iluminar as discrepâncias entre o desenho original e as contingências locais ${ }^{24}$. Esta revisão da lógica avaliativa tradicional contribui para que as intervenções não sejam vistas como variáveis independentes do contexto local. Na maioria das vezes, na avaliação de programas e políticas públicas a preocupação maior é com o grau de execução das atividades previstas como, por exemplo, o número de consultas realizadas, medicamentos dis- tribuídos, encontros com as famílias ou reuniões com as associações civis. Ainda que tais dados sejam relevantes, em geral, não expressam como estão sendo geradas as respostas ao programa. Sobretudo no caso dos programas e iniciativas intersetoriais e participativas é preciso entender como operam os mecanismos de mudança previstos. Como analisa Weiss ${ }^{25}$, no caso de um programa voltado à redução do número de mulheres grávidas, o aconselhamento sobre métodos contraceptivos na perspectiva avaliativa tradicional é visto como atividade meio para atingir as metas previstas. No entanto, explorando concomitantemente a teoria do programa e seus mecanismos de ação, o foco é deslocado para o conhecimento gerado nos encontros, para analisar os diferentes graus de confiança que as mulheres ganharam para "negociar" o uso de contraceptivos com seus parceiros ou ainda para perceber os possíveis tabus sobre planejamento familiar existentes no contexto local.

No caso dos programas de educação nutricional, por exemplo, para além da sistematização do número de encontros realizados, interessa saber como as questões foram abordadas, quais foram as possibilidades e os limites para compreender e interpretar os conteúdos e de que maneira o conteúdo foi ou não integrado ao cotidiano das práticas alimentares locais. Em um programa de merenda escolar, além da identificação do número de refeições distribuídas ou de crianças atendidas, é crucial compreender como os alimentos estiveram inseridos no contexto cultural e social dos alunos, quais foram as demandas que dificultaram o processo ou, ainda, quais foram os novos recursos introduzidos e que facilitaram a aceitação dos alimentos.

A preocupação com a teoria e os mecanismos de ação ${ }^{26}$ dos programas de segurança alimentar e nutricional torna possível compreender os processos através dos quais serviços e bens são ofertados esclarecendo como os efeitos são alcançados, quais são as consequências não previstas da intervenção e como são sustentadas as conexões entre os modelos causais adotados, os processos e os resultados. Com isso, é possível analisar a validade das concepções que ligam processos e resultados nos diferentes programas e iniciativas públicas.

Para Weiss ${ }^{25}$ e Chen $^{27}$ as teorias que orientam as intervenções não são necessariamente corretas ou consensuais, ao contrário, podem revelar ambiguidades e contradições no que se refere à compreensão dos problemas a serem enfrentados ou dos possíveis efeitos perversos das estratégias ado- 
tadas. No desenho avaliativo do programa, porém, tais questões devem ser privilegiadas a fim de ampliar o olhar para o processo de tradução dos objetivos em atividades na dinâmica operacional de cada intervenção. A avaliação baseada na combinação entre teoria do programa e análise do processo de implementação além de contribuir para iluminar os alvos de mudança também ajuda a indicar caminhos para a coleta de dados especialmente relevantes. Evitando a oposição entre dados quantitativos e qualitativos ou entre diferentes técnicas e procedimentos metodológicos, a perspectiva é agregar métodos de maneira plural. Entrevistas, observação local, dados quantitativos e técnicas estatísticas podem, desta maneira, ser articuladas em um mesmo desenho avaliativo. A visão da avaliação de programas como uma rota pré-determinada aplicável de maneira mecânica a qualquer iniciativa e em qualquer contexto é abandonada na medida em que um mesmo mecanismo de ação pode ser reinterpretado pelos agentes locais e contribuir para resultados opostos em contextos mais ou menos favoráveis ${ }^{28}$.

No caso de programas de transferência de renda voltados à população vivendo em situação de insegurança alimentar e nutricional a premissa teórica básica é de que o benefício monetário garante maior acesso e consumo de alimentos saudáveis. No entanto, a iniciativa pode resultar no aumento do consumo de alimentos ricos em açúcares, gorduras ou de produtos enlatados ${ }^{29}$. Isto mostra que práticas alimentares mais saudáveis não ocorrem imediatamente a partir do aumento da renda, outras ações são necessárias de acordo com a dinâmica de cada contexto. Em algumas regiões, a introdução de novos alimentos nas cantinas escolares, o reconhecimento das fronteiras simbólicas e culturais em jogo ou a associação da transferência de renda a práticas educativas pode ampliar o alcance dos programas.

Programas de estímulo à agricultura familiar também trazem importantes aprendizados. Em geral, a teoria é sustentada pela visão de que ao elevar o preço pago aos agricultores torna-se possível enfraquecer o circuito de exploração dos atravessadores, escoar a produção e contribuir para a melhoria da qualidade e quantidade dos alimentos comercializados no contexto local. No entanto, no processo de implementação, caso o governo não garanta o pagamento sem atrasos os pequenos produtores desprovidos de poupança prévia podem preferir a venda de suas mercadorias aos atravessadores e, desta forma, evitar o risco de ter que esperar 30 ou 60 dias pelos recursos financeiros ${ }^{30}$.
Assim, compreender onde o programa fracassa, quais são os conflitos, constrangimentos e obstáculos não previstos é tão importante quanto identificar resultados positivos. Como analisam Pawson e Tilley ${ }^{31}$, os programas podem ser introduzidos em contextos que alimentam ou sustentam os problemas que se quer atacar. Segundo os autores, a principal tarefa avaliativa é, portanto, analisar quais são as condições sociais e culturais necessárias para que a teoria e os mecanismos de ação do programa operem e como eles estão presentes em cada contexto.

\section{Conclusões}

A segurança alimentar e nutricional não é uma questão isolada ou um privilégio para poucos ${ }^{32}$. Ao contrário, é um tema fortemente articulado à saúde, ao ambiente e ao desenvolvimento sustentável global. A violência da fome, o aumento da obesidade, o esgotamento das fontes de energia renováveis, a redução dos recursos hídricos e as ameaças à biodiversidade são faces de uma dinâmica política e econômica cujos impactos transcendem grupos ou fronteiras regionais. No contexto recente, as mudanças climáticas e as crises econômicas tendem a revelar de maneira ainda mais contundente a necessidade de soluções sistêmicas. Mas, ao mesmo tempo, dificilmente será possível encontrar respostas e desenhar novas estratégias de intervenção sem o estudo das especificidades e singularidades locais.

Padrões de desenvolvimento equitativos e sustentáveis envolvem prerrogativas e direitos substantivos para que os indivíduos tenham acesso permanente a alimentos saudáveis sem ameaçar a biodiversidade, o abastecimento futuro e a qualidade de vida das novas gerações. Nesta perspectiva, é preciso enfrentar desafios e avançar na consolidação de novos processos avaliativos a fim de gerar evidências de efetividade das ações e também aprendizados para a construção de alternativas. A avaliação centrada nos resultados finais dos programas não informa como tomadores de decisão e gestores devem conduzir o processo de implementação das ações, rever as estratégias adotadas e corrigir problemas. Através da pesquisa empírica sistemática na área, ênfase em estudos de caso e interação entre dados quantitativos e qualitativos oriundos do processo de implementação local é possível captar a complexidade dos programas de segurança alimentar e nutricional e contribuir para aprendizados e mudanças efetivas. 


\section{Referências}

1. Castro J. Geografia da Fome. Rio de Janeiro: Livraria-Editora da Casa do Estudante do Brasil; 1953.

2. Maluf RS. Segurança alimentar e nutricional. Rio de Janeiro: Ed. Vozes; 2007.

3. Brasil. Conselho Nacional de Segurança Alimentar e Nutricional (CONSEA). II Conferência Nacional de Segurança Alimentar e Nutricional. Relatório Final. Brasília: CONSEA; 2004. [Internet]. [acessado 2012 set 10]. Disponível em www.planalto.gov.br/ consea

4. Brasil. Decreto-Lei no 11.346 , de 15 de setembro de 2006. Cria o Sistema Nacional de Segurança Alimentar e Nutricional. SISAN com vistas em assegurar o direito humano à alimentação adequada e dá outras providências. Diário Oficial da União 2006; 18 set.

5. Lang T, Heasman M. Food Wars - The global battle for mouths, minds and markets, London: Earthscan; 2004.

6. Lang T, Heasman M. The war of paradigms: time for a new framework?. In: Food Wars - The global battle for mouths, minds and markets. London: Earthscan; 2004. p. 16

7. Agência Nacional de Vigilância Sanitária (ANVISA). Programa de Análise de Resíduo de Agrotóxico em Alimentos (PARA). Dados da coleta e análise de alimentos de 2010. ANVISA. [Internet]. [acessado 2012 jul 15]. Disponível em: www.anvisa.gov.br

8. Associação Brasileira de Saúde Coletiva (Abrasco). Dossiê: um alerta sobre os impactos dos agrotóxicos na saúde. Parte 1-Agrotóxicos, Segurança Alimentar e Saúde, 2012. [acessado 2012 ago 10]. Disponível em: www.abrasco.org.br

9. Brasil. Lei $n^{\circ} 7.802$ de julho de 1989. Lei Federal dos Agrotóxicos. Diário Oficial da União 1989; 12 jul.

10. Almeida D. Agricultura urbana e segurança alimentar em Belo Horizonte: cultivando uma cidade sustentável. Rev Agriculturas: experiências em agroecologia 2004; 1:25-28.

11. Brasil. Ministério do Desenvolvimento Social e Combate à Fome (MDS). Programa de Aquisição de Alimentos (PAA). Brasília: MDS; 2006. [Internet]. [acessado 2011 mar 18]. Disponível em: http:/ /www.mds.gov.br

12. Hernadez Contreras J. Patrimônio e Globalização: o caso das culturas alimentares. In: Canesqui AM, Garcia RWD, organizadores. Antropologia e Nutrição. Rio de Janeiro: Editora Fiocruz; 2005. p. 129146.

13. American Heart Association (AHA). Food Advertising and Marketing to Children. Washington. [Internet]. [acessado 2014 mar 27]. Disponível em: http:/ /www.heart.org/advocacy

14. Mikkelsen L, Merlo C, Lec V, Chao C. "Where's the fruit? Fruit content of the most highly advertised children's food and beberages. The Prevention Institute 2007. [Internet]. [acessado 2014 mar 27]. Disponível em: http://www.preventioninstitute.org/ component/jlibrary/article/id-56/127.html
15. Brasil. Consulta Pública $\mathrm{n}^{\circ} 71$, de 10 de Novembro de 2006. Proposta de Regulamento Técnico sobre oferta, propaganda, publicidade, informação e a outras práticas correlatas cujo objeto seja a divulgação ou promoção de alimentos com quantidades elevadas de açúcar, de gordura saturada, de gordura trans, de sódio e de bebidas com baixo teor nutricional, quaisquer que sejam as formas e meios de sua veiculação. Diário Oficial da União 2006; 13 nov.

16. Monteiro CA, Castro IR. Porque é necessário regulamentar a publicidade de alimentos. Rev Cienc e Cultura 2009; 61(4):56-59.

17. Prado S, Gugelmin SA, Mattos RA, Silva JK, Olivares PSG. A pesquisa sobre segurança alimentar e nutricional no Brasil de 2000 a 2005: tendências e desafios. Cien Saude Colet 2009; 15(1):7-18.

18. Mc Queen D. Evidence and theory: continuing debates on evidence and effectiveness. In: Mc Queen D, Jones C, editors. Global Perspectives on Health Promotion Effectiveness. New York: Springer; 2007. p. 281-303.

19. L'Abbate S. As políticas de alimentação e nutrição no Brasil, I: Período de 1940 a 1964. Rev Nutrição 1988; 1(2):87-138.

20. Santos SMC, Santos LMP. Avaliação de políticas públicas de segurança alimentar e combate à fome no período de 1995-2002: abordagem metodológica. Cad Saude Publica 2007; 23(5):1029-1040

21. Burlandy L, Magalhães R, Maluf R. Construção e promoção de sistemas locais de segurança alimentar e nutricional: aspectos produtivos, de consumo, nutricional e de politicas públicas. Rio de Janeiro: CPDA/ UFRRJ; 2006. (Série Relatórios Técnicos, n.3).

22. Bodstein R. The complexity of the discussion on effectiveness and evidence in health promotion practices. Promotion \& Education 2007; 1(Supl.):16-20.

23. Potvin L, Haddad S, Frolich KL. Beyond Process \& Outcome Evaluation: a Comprehensive Approach for Evaluating Health Promotion Programmes. In: Rootman I, Goodstadt M, Hyndman B, McQueen DV, Potvin L, Springett J, Ziglio E, editors. Evaluation in Health Promotion: Principles \& Perspective. Geneva: WHO Regional Publications; 2001. p. 4562. (European Series, n. 92)

24. Champagne F, Contrandiopoulos AO, Brousselle A, Hartz Z, Denis J. L'évaluation dans le domaine de la santé: concepts et methods. In: Brousselle A, Champagne F, Contrandiopoulos AO, Hartz Z. L'évaluation: concepts et methods. Montréal: Les Presses de l'Université de Montreal; 2009. p. 35-56.

25. Weiss C. Evaluation. New Jersey: Prentice Hall; 1998.

26. Pawson R. Nothing as practical as a good theory. Evaluation 2003; 9(4):471-490

27. Chen TH. Theory-driven Evaluations. London: Sage Publications; 1990.

28. Chen HT, Rossi P. Issues in the theory-driven perspective. Evaluation and program planning 1989; 12(4):299-306.

29. Instituto Brasileiro de Análises Sociais e Econômicas (Ibase) Repercussões do Programa Bolsa Família na segurança alimentar e nutricional: relatório síntese. Rio de Janeiro: Ibase; 2008. 
30. Vaitsman J, Paes-Souza R, organizadores. Avaliação de Politicas e Programas do MDS. Brasília: Secretaria de Avaliação do Desenvolvimento Social e Combate à Fome; 2007.

31. Pawson R, Tilley N. Realistic Evaluation. London: Sage Publications; 1997.

32. Bosi ML. Desafios na interface pesquisa-construção do direito humano à alimentação: reflexões no âmbito da segurança alimentar e nutricional. Cien Saude Colet 2010; 15(1):22-24.

Artigo apresentado em 19/07/2013

Aprovado em 30/11/2013

Versão final apresentada em 11/12/2013 